\title{
Theranostic body fluid cleansing: rationally designed magnetic particles enable capturing and detection of bacterial pathogens $\dagger$
}

\author{
M. Lattuada $\ddagger^{\mathrm{a}}$ Q. Ren $\ddagger^{\mathrm{b}}$ F. Zuber, ${ }^{\mathrm{b}}$ M. Galli, ${ }^{\mathrm{c}}$ N. Bohmer, ${ }^{\mathrm{b}}$ M. T. Matter, ${ }^{\mathrm{b}}$ \\ A. Wichser, ${ }^{b}$ S. Bertazzo, ${ }^{d}$ G. B. Pier ${ }^{e}$ and I. K. Herrmann*b
}

\begin{abstract}
We report on theoretical and experimental considerations on bacteria capturing and enrichment via magnetic separation enabling integrated diagnosis and treatment of blood stream infections. We show optimization of carrier-pathogen interactions based on a mathematical model followed by an experimental proof-of-concept study along with investigations on the process safety.
\end{abstract}

Sepsis is a severe, potentially life-threatening medical condition. ${ }^{1}$ Despite major technological advances in recent years, sepsis mortality remains disturbingly high. The management of sepsis patients is technically challenging and costly, with only a few therapeutic options available. ${ }^{2}$ Early diagnosis and identification of the microbe causing this sepsis has a major impact on the patient's outcome. ${ }^{3}$ However, diagnosis remains challenging as most sepsis patients present with clinical symptoms indistinguishable from those who suffer from non-infectious systemic inflammatory response syndrome (SIRS). ${ }^{1,4}$ One of the major problems in diagnostics is the typically extremely low bacterial concentration in human sepsis patients $(<10$ colony forming units (cfu) per $\mathrm{mL}$ ), leading to long blood culture times and high numbers of false negatives. ${ }^{5}$ Diagnostic uncertainty, in conjunction with the direct dependence of patient outcome on early antibiotic treatment, has led to an overuse of antibiotics. Treating all patients who show SIRS symptoms without a proper diagnosis not only increases costs, but leads to other complications and increased microbial resistance, which is equally undesirable.

\footnotetext{
${ }^{a}$ Adolphe Merkle Institute, University of Fribourg, Fribourg, Switzerland

${ }^{b}$ Department Materials Meet Life, Swiss Federal Laboratories for Materials Science and Technology (Empa), Lerchenfeldstrasse 5, 9014 St. Gallen, Switzerland. E-mail: inge.herrmann@empa.ch; Tel: +41587657153

'Dipartimento di Chimica, Università degli Studi di Milano, Via Golgi 19, 20133 Milano, Italy

${ }^{d}$ Department of Medical Physics and Biomedical Engineering,

University College London, Malet Place Engineering Building, London, UK

${ }^{e}$ Brigham and Women's Hospital, Harvard Medical School, 181 Longwood Avenue, Boston, MA 02115, USA

$\dagger$ Electronic supplementary information (ESI) available. $\ddagger$ Contributed equally.
}

Magnetic blood purification has recently been suggested as a treatment modality in sepsis by us and others. ${ }^{6-10}$ In magnetic separation-based blood purification, magnetic particles functionalized with capturing moieties bind to pathogens and are subsequently removed from the blood by magnetic separation. Magnetic blood purification is especially beneficial if highmolecular weight compounds or cells need to be cleansed; as such compounds are insufficiently removed by current blood purification systems, such as hemofiltration or hemosorption. Utilizing freely diffusing, spherical particles offers superior surface accessibility and hence speeds up the binding process substantially compared to traditional processes. The feasibility of the process has been demonstrated in recent years in various in vitro and in vivo settings by us and others. ${ }^{6-12}$ However, clinical translation is currently hampered by safety and specificity issues of the magnetic capturing agents. One of the most critical limitations of the current magnetic particle based approaches is that the magnetic beads need to be functionalized with target-specific moieties. ${ }^{10}$ However, particularly in medical emergency situations, such as sepsis, the causative pathogen is usually not known. Therefore, there is an urgent clinical need for capturing moieties that bind to a broad range of relevant pathogens. Engineered mannose binding-lectin (MBL) protein has recently been successfully applied to bind to a range of pathogens, and the target binding and separation efficacy has been investigated as a function of bead size by Kang and colleagues. ${ }^{10,13}$ Another important challenge to be addressed is associated with the safe use of magnetic particle-based capturing agents. It remains to be demonstrated whether particles can be removed completely from blood before the blood is recirculated into the organism, and if not, the risks of particles escaping the separation need to be assessed comprehensively. For example, we have recently demonstrated in a long-term exposure study that highly magnetic carbon-encapsulated iron carbide nanoparticles can still be found in the resident macrophages of the liver and the lung even after prolonged exposure times of up to one year in mice. ${ }^{14}$ It is thus favorable to use biologically degradable ironoxide based carriers; however, this comes at the cost of reduced 
magnetization and decreased capturing efficacy. ${ }^{15}$ Surprisingly, and despite the decade-long history of magnetic blood purification, it remains largely unclear how to achieve optimal target isolation at acceptable risk-benefit ratios, hence precluding effective clinical translation of a potentially game-changing therapy for sepsis patients.

Here, we performed theoretical and experimental studies to investigate whether a newly developed human IgG1 monoclonal antibody against poly- $N$-acetylglucosamine (PNAG), ${ }^{16}$ which binds to all but one of the pathogens responsible for the 10 most frequent nosocomial infections, can be immobilized on magnetic beads in order to efficiently capture and detect pathogenic bacteria without the need for prior pathogen identification. We demonstrate quantitative capturing efficacies and degradation of the beads under physiological conditions, both being critical parameters for effective translation of the approach into clinical settings.

First, we developed a mathematical model to elaborate upon the benefits of a magnetic particle-based blood purification process over traditional blood purification systems for capturing bigger target entities, such as bacteria. This model is utilized to work out intrinsic process limitations and optimal conditions for magnetic blood cleansing as a function of particle size and concentration as well as target size and concentration. The required contact times for a binding event to happen determine the efficacy and throughput of an extracorporeal magnetic blood purification process. Given that the human blood volume is about $5 \mathrm{~L}$ and that the whole blood volume should preferably be processed in a few hours (in analogy to dialysis based processes) with a total extracorporeal volume of less than $300 \mathrm{~mL}$ at a given time, the contact times between magnetic particles and blood should be in the order of seconds to minutes. In order to estimate the contact times required for efficient target capturing as a function of target size, we developed a model for the calculation of (i) the adsorption of small molecules on the surface of magnetic particles and, in analogy, (ii) the number of magnetic particles adsorbed on the surface of bacteria. Both the particles and the bacteria are modeled as spheres. First, we calculated the time required to remove small target molecules, such as ions, proteins or bacterial toxins, using a magnetic bead number concentration of $10^{10}$ per mL. The contact times required for both small molecule targets $(1 \mathrm{~nm}, 1 \mu \mathrm{M}$ concentration range) and medium sized targets, such as endotoxins, (10 nm, $\mathrm{nM}$ concentration range) are well below 1 minute, which is in good agreement with previously reported experimental findings (Fig. 1a). ${ }^{6,11}$ As small molecules are capable of diffusing inside of porous adsorbents (such as porous activated carbon, etc.), the use of particle based adsorbents is not absolutely necessary and small molecules and ions are removed at a sufficiently high efficiency via traditional blood purification processes, such as hemodialysis, hemofiltration or hemosorption. ${ }^{17}$ For bigger molecules and cells, however, the surface area of pores remains inaccessible and the external surface area of an adsorbent becomes the relevant parameter, hence the use of spherical particles provides considerable competitive advantages over traditional adsorbents. The external surface becomes a critical parameter dictating the overall throughput and efficacy of the process. Thus, we first calculated the time-dependent evolution of the number concentration of bacteria (Staphylococcus aureus, $1 \mu \mathrm{m}, 10^{4}$ colony-forming units (cfu) per $\mathrm{mL}$ ) as a function of particle number concentration. It becomes evident that when using $300 \mathrm{~nm}$ beads, there are no unbound bacteria left after 180 seconds (Fig. 1b). After 10 minutes, every bacterium is bound by 10 or more magnetic beads (Fig. 1c). Most importantly, calculations also show that particles are in excess for most clinically relevant bacterial concentrations (Fig. 1d, for bacteria concentrations from $10-10^{4} \mathrm{cfu}$ per $\mathrm{mL}$ ). Taken together, these calculations show that there is a window of opportunity where reasonable binding of bacteria can be achieved by reasonable concentrations of magnetic beads $\left(10^{10}-10^{12}\right.$ particles per $\left.\mathrm{mL}\right)$ in clinically relevant times (few minutes). Additionally, these calculations demonstrate that capturing times are critically dependent on the particle number and increase to less appealing minimal contact times ( $\gg 10$ minutes) for particle number concentrations of $10^{9}$ per $\mathrm{mL}$ or less, significantly limiting the process throughput. Importantly, these calculations also show that while for small molecules traditional blood purification systems work well, the binding of bacteria and larger targets becomes inefficient as conventional systems are limited by low surface accessibility (typically equivalent to $10^{8}$ to $10^{9}$ particles per $\left.\mathrm{mL}\right){ }^{18}$

In the next step, we sought to demonstrate experimentally that bacteria can indeed be bound within reasonable time frames using particle concentrations of $10^{10}$ per $\mathrm{mL}$ of $300 \mathrm{~nm}$ iron oxide based beads. In order to do that, we assembled magnetic particle based capturing agents using magnetite-based nanoclusters with a size of $300 \mathrm{~nm}$, a saturation magnetization of 40 emu per $\mathrm{g}$ and a specific surface area of $10 \mathrm{~m}^{2}$ per $\mathrm{g}$. We attached fully human IgG-based PNAG antibodies to protein A/G-hybrid coated magnetic iron oxide nanoclusters consisting of $10 \mathrm{~nm}$ iron oxide primary particles (Fig. 2a and b). Measurement of the hydrodynamic particle size in physiological medium via Nanoparticle Tracking Analysis (NTA) show narrow particle size distributions centered around $300 \mathrm{~nm}$ before and after functionalization, indicative of the reasonable colloidal stability of the particle dispersions (Fig. 2c). Particle number concentration measurements were also performed on the NTA using diluted dispersions in order to adjust particle number concentrations for subsequent experiments. Carbon, nitrogen, and hydrogen measurements further confirmed the successful binding of the PNAG-antibody to the particle surface. The colloidal stability of the functionalized particle suspensions in protein-rich physiological media (PBS + $1 \%$ serum) was further analyzed by measuring size distributions and the zeta potential of the functionalized particles, yielding a mean average diameter of $366 \pm 2 \mathrm{~nm}$ in phosphate buffered saline (PBS) and $385 \pm 2 \mathrm{~nm}$ in serum-containing PBS and zeta potential values of $-20 \mathrm{mV}$ and $-17 \mathrm{mV}$ in serum-free and serum-containing PBS, respectively (Fig. 2d-f).

Magnetic particles functionalized with protein A/G and with or without the PNAG antibody, respectively, were then dispersed in PBS at a concentration of $5 \mathrm{mg}$ (particle mass) per $\mathrm{mL}$ and further diluted for the experiments. In order to demonstrate the 
a)
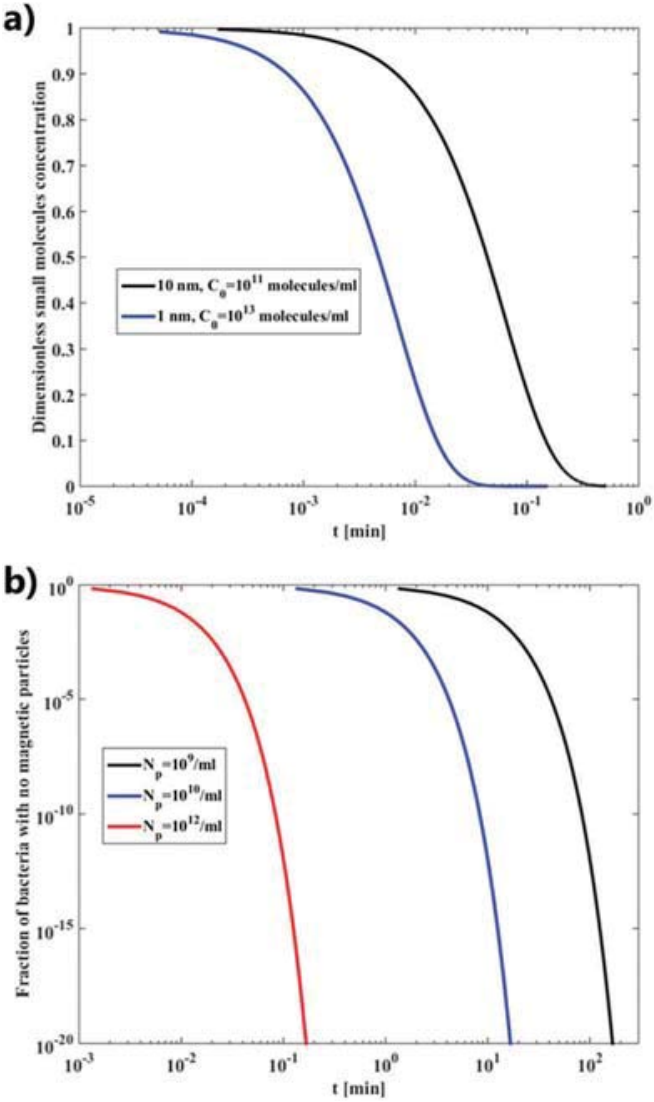

c)

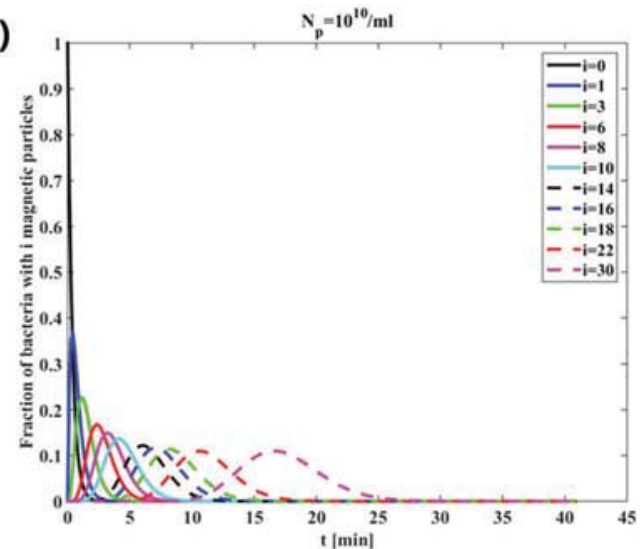

d)

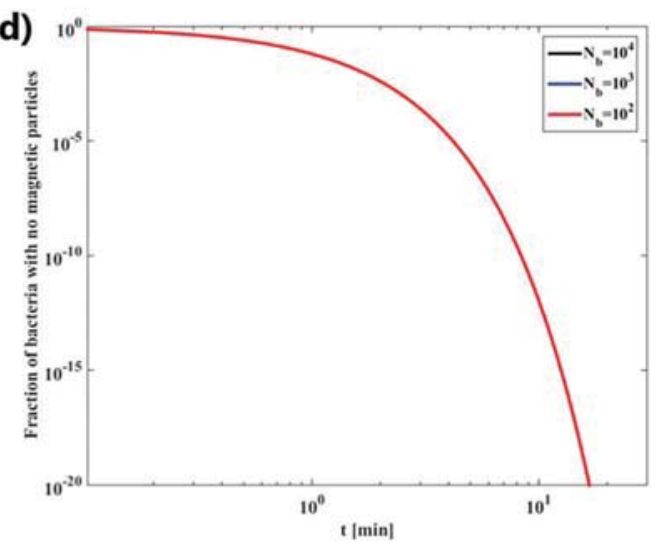

Fig. 1 Simulations of magnetic particle-pathogen binding. Time evolution of unbound small molecules with a size of 1 and $10 \mathrm{~nm}$, in the nanomolar and micromolar concentration range, respectively (a). Time evolution of bacteria with no bound particles, as a function of particle concentration $\left(N_{\mathrm{p}}\right)(\mathrm{b})$. Time evolution of the number of particles attached to bacteria for a bacteria concentration of $10^{4}$ per $\mathrm{mL}$ and a particle number concentration of $10^{10}$ per $\mathrm{mL}$. The bacteria number concentration has been normalized by their initial concentration (c). Time evolution of bacteria with no bound particles, as a function of bacteria concentration $\left(N_{\mathrm{b}}\right)(\mathrm{d})$.

feasibility of the assembled agent for capturing bacteria, we first confirmed that the beads can be recovered quantitatively through magnetic separation. While the efficiency of the separation at a contact time of 10 seconds was only $57.10 \%$, it increased at higher separation times, achieving $91.81 \%$ separation at 1 minute and $99.87 \%$ separation at 5 minutes. At a separation time of 20 minutes, the iron signal was indistinguishable from the background level of PBS (Fig. 2g).

Bacteria capturing was further investigated with the aim of demonstrating the theranostic potential of the approach (Fig. 3a). For capturing bacteria, samples containing pathogenic bacteria (S. aureus, ATCC 6538) at various concentrations $\left(10^{2}, 10^{3}, 10^{4}\right.$ bacteria per $\mathrm{mL}$ ) were prepared in physiological media. Particles at a concentration of $10^{10}$ particles per $\mathrm{mL}$ were subsequently added to the bacteria contaminated samples. After 10 minutes, the particles were separated from the supernatant by placing a $1 \mathrm{~cm}^{3}$ neodymium permanent magnet $(1.37 \mathrm{~T})$ next to the sample tube. Bacteria quantification in the supernatant and on the particle surface via plating and optical absorption revealed that the bacteria were efficiently captured by the anti-PNAGfunctionalized beads (capturing efficacy $>98 \%$, Fig. $3 \mathrm{~b}$ ). In contrast, bacteria capturing by the protein A/G-functionalized beads (control) without antibody was significantly less efficient $(<40 \%)$. Next, the theranostic potential was demonstrated by subjecting the pathogen-loaded beads to quantitative polymerase chain reaction (qPCR) based identification of the bacterial strain. Indeed it was possible to detect and identify S. aureus on the beads via qPCR (Fig. 3c). Interestingly, the bacteria showed significant re-growth on the beads, which could potentially speed up blood culture and has theranostic relevance. The re-growth of bacteria enriched on the beads is significantly more pronounced compared to the untreated supernatant (see the ESI, $\dagger$ Fig. S1). The captured bacteria can further be visualized using electron microscopy despite the fact that magnetic beads were present in large excess (as expected, based on the modelling data, Fig. 1d) (Fig. 3d).

In order to be appealing for clinical application, however, safety concerns have to be addressed. To that end, we demonstrated that there is no detectable effect on cell viability even after prolonged contact of up to 24 hours for the particle concentrations used $\left(10^{10}\right.$ particles per $\left.\mathrm{mL}\right)$ (Fig. 3e). We also demonstrated that the uptake of particles into monocytes (THP-1 cells) is very limited within the intended contact times (10 minutes or less). Measurement of the side scattering intensity using flow cytometry showed a time-dependent increase indicative of particle uptake (Fig. 3f). While a minor 
a)
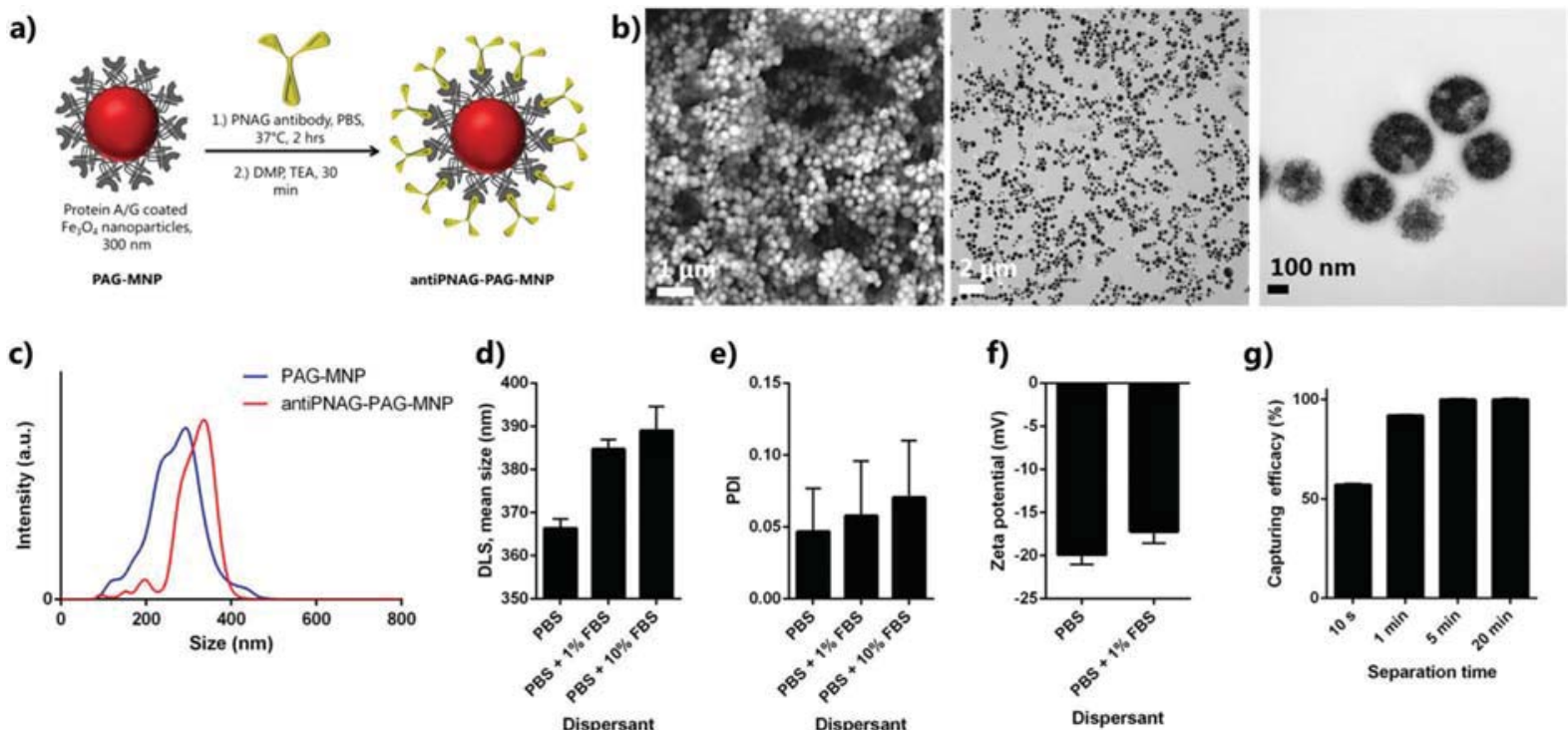

g)

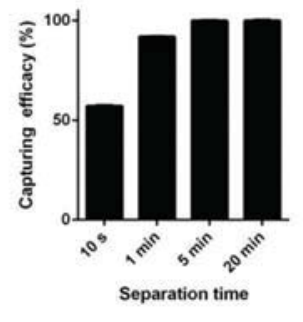

Fig. 2 Magnetic particle-based capturing agents were assembled using protein A/G-coated Fe ${ }_{3} \mathrm{O}_{4}$ (PAG-MNP) nanoclusters and a human antibody against poly- $N$-acetylglucosamine (PNAG) (a). Scanning and transmission electron micrographs of the functionalized antiPNAG-PAG-MNP nanoclusters (b). The hydrodynamic size of the $\mathrm{Fe}_{3} \mathrm{O}_{4}-\mathrm{PAG}$ nanoclusters before and after antibody-functionalization measured in phosphate-buffered saline (PBS) using nanoparticle tracking analysis (NTA) (c). Mean size (d), polydispersity index (e) and zeta potential (f) of beads suspended in phosphate buffered saline (PBS), and PBS supplemented with $1 \%$ and $10 \%$ fetal bovine serum (FBS), respectively, measured using dynamic light scattering (DLS). Bead capturing efficiency from suspension measured using Inductively Coupled Plasma Mass Spectroscopy (ICP-MS) as a function of separation time (g).

shift in the side scatter intensity is observed 10 minutes after incubation, it subsequently increases and at 24 hours a considerable shift is seen that is indicative of significant particle uptake into human blood monocytic cells. Importantly, particles are eventually degraded over time at $\mathrm{pH}$ conditions comparable to lysosomal pH (Fig. 3g).

This proof of principle study demonstrates for the first time the theranostic potential of a magnetic separation-based body fluid purification approach. Bacteria can be captured from suspension and can subsequently be identified on the magnetic beads. This study forms the groundwork for a theranostic process where the bacterial load can be reduced and bacterial spread can be limited. Meanwhile, magnetic beads loaded with bacteria can be recovered from the separator and subjected to diagnostic analysis, hence decreasing both the time to diagnosis (as compared to blood culture due to bacteria enrichment) and the time to therapeutic administration of the appropriate antibiotics. While the therapeutic efficacy of pathogen removal remains to be demonstrated in clinics, the proposed theranostic approach is sought to help reduce the overuse of antibiotics by allowing rapid bacteria identification and identification of resistance genes. In future it may at the same time provide a treatment modality to bridge the time to diagnosis when appropriate antibiotic therapy can be administered.

\section{Materials and methods}

\section{Mathematical model}

A population balance equation was used in order to compute the change in concentration of the magnetic particles with time, as well as the time evolution of the fraction of bacteria with a given number of magnetic particles attached to them. The mass balance for the concentration $N_{\mathrm{MP}}$ of magnetic particles:

$$
\frac{\mathrm{d} N_{\mathrm{MP}}}{\mathrm{d} t}=-N_{\mathrm{MP}} \sum_{i=0}^{M} K_{\mathrm{D}, i}(t) N_{\mathrm{B}, i}
$$

where $N_{\mathrm{B}, i}$ is the number concentration of bacteria containing $i$ magnetic particles, and $M$ is the maximum number of magnetic particles per bacteria. When this number of particles is bound to a bacterium, its surface is fully covered by particles, preventing other particles from binding. Even the kinetic constant differs for all particles, since its degree of surface coverage depends on the number of particles attached to its surface. The corresponding population balance equations for the bacteria are the following:

$$
\frac{\mathrm{d} N_{\mathrm{B}, i}}{\mathrm{~d} t}=K_{\mathrm{D}, i-1}(t) N_{\mathrm{MP}} N_{\mathrm{B}, i-1}-K_{\mathrm{D}, i}(t) N_{\mathrm{MP}} N_{\mathrm{B}, i}
$$

The binding constant is provided by Smoluchowski's law for a diffusion-limited process, modified to take into account the amount of surface of the bacteria not entirely accessible:

$$
K_{\mathrm{D}}(t)=\frac{2 k_{\mathrm{B}} T}{3 \eta}\left(R_{\mathrm{B}}+R_{\mathrm{MP}}\right)\left(\frac{1}{R_{\mathrm{B}}}+\frac{1}{R_{\mathrm{MP}}}\right) \alpha(t)
$$

The corresponding value of $a_{i}(t)$ is given by the following equation:

$$
\alpha_{i}(t)=\left(1-\frac{i \cdot R_{\mathrm{MP}}{ }^{2}}{4 \varepsilon\left(R_{\mathrm{MP}}+R_{\mathrm{B}}\right)^{2}}\right)
$$

In this case, it is necessary to solve $M+1$ eqn (2), in addition to eqn (1). This approach allows the calculation of the average number of particles per bacteria, and to estimate how many bacteria do not have any particles attached to them, as a function of time. 
a)

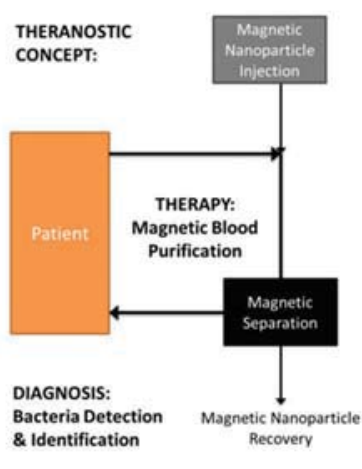

b)

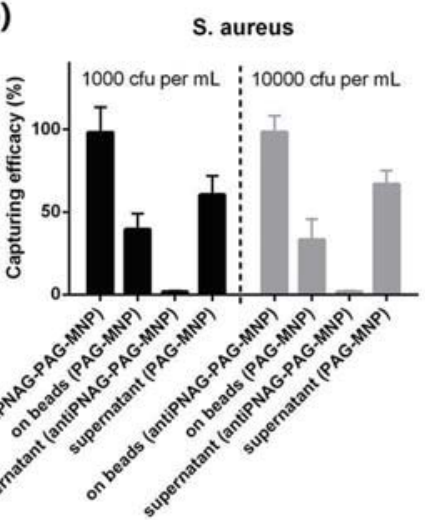

c)

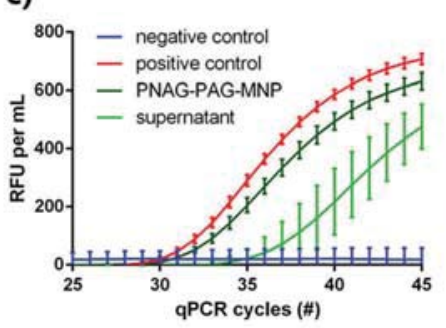

d)

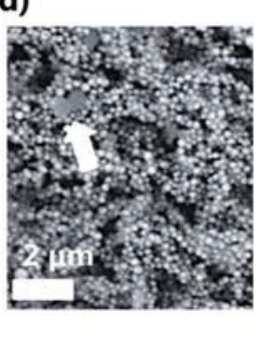

e)

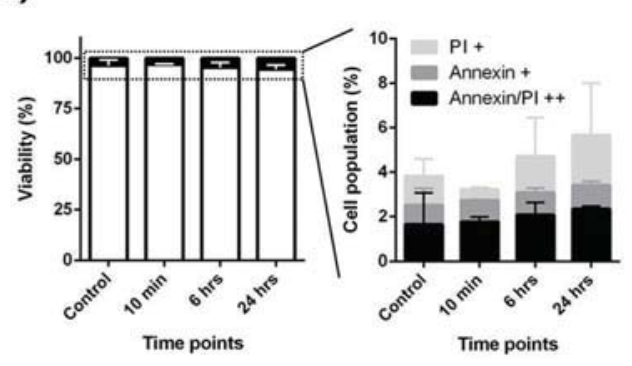

f)

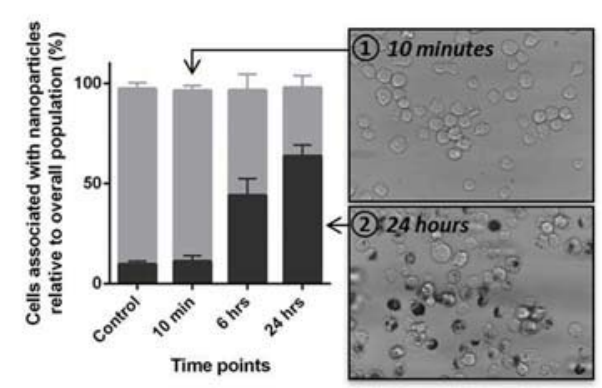

g)

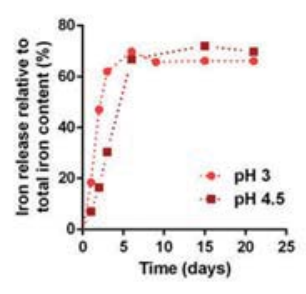

Fig. 3 Theranostic concept: body fluid is subjected to magnetic blood purification where circulating pathogens are removed (therapeutic benefit). Magnetic beads loaded with bacteria can then be recovered from the separator and subjected to diagnostic analysis (a). Bacteria removal efficiency for S. aureus at two different bacteria concentrations $\left(10^{3}\right.$ and $10^{4} \mathrm{cfu}$ per $\mathrm{mL}$ ) and a nanoparticle number concentration of $10^{10}$ per $\mathrm{mL}$ (b). Quantitative polymerase chain reaction (qPCR) detection of bacteria in the supernatant and on the beads (c). Scanning electron micrograph showing the particle pellet after separation with bacteria captured by magnetic particles (arrow), scale bar $=4 \mu \mathrm{m}$ (d). Monocyte viability after 24 hour exposure to the relevant particle concentrations $\left(10^{10}\right.$ particles per $\mathrm{mL}, 5 \times 10^{5}$ monocytes per $\mathrm{mL}$ ) (e). Particle uptake into monocytes measured by the increase in granularity (side scattering) in flow cytometry at different contact times, and the corresponding light microscopy images of monocytes incubated with particles for 10 minutes and 24 hours, respectively (f). Particle degradation over time in citrate buffer at $\mathrm{pH} 3.0$ and $\mathrm{pH} 4.5$ (g).

Magnetic particles were modeled as spheres with a diameter of $300 \mathrm{~nm}$ and bacteria with a diameter of $1 \mu \mathrm{m}$. The number concentration of particles per $\mathrm{mL}$ is $10^{9}, 10^{10}$ and $10^{12}$. The differential eqn (2) has been solved using Matlab $^{\circledR}$ (Mathworks) ODE solver ode15s.

\section{Magnetic carrier assembly}

Magnetic capturing agents were assembled using nanoclusters functionalized with a recombinant fusion protein $\mathrm{A} / \mathrm{G}$ (PAG, Bio-Adembeads PAG, Ademtech, Pessac, France). The PAG lacks albumin-binding domains, cell wall binding regions and other non-specific binding domains. Magnetic beads were washed thoroughly $(3 \times)$ with PBS containing 0.65\% Tween 20 (pH 7.5). Then, fully human PNAG monoclonal antibody (obtained from Prof. G. Pier, Harvard University) was added in PBS $(1 \mu \mathrm{g}$ per $10 \mu \mathrm{L}$ beads) and incubated for 1 hour at room temperature on a linear shaker. Then, the unbound antibody was removed by washing the beads three times in PBS containing 0.65\% Tween 20 ( $\mathrm{pH} 7.5$ ). Where applicable, the beads were resuspended in $200 \mathrm{mM}$ triethanolamine containing $20 \mathrm{mM}$ dimethyl pimelimidate dihydrochloride (DMP). The beads were then thoroughly washed and resuspened in PBS at a concentration of $10^{11}$ particles per $\mathrm{mL}$. Particle size measurements were made in buffer using a Nanoparticle Tracking Analysis (NTA) device (NS500, Nanosight) and a
ZetaSizer $\left(90^{\circ}\right.$ configuration). The zeta potential was measured on a ZetaSizer in $0.1 \times$ PBS (pH 7.4).

\section{Particle capturing efficacy}

In order to quantify the separation efficiency under a magnetic field, a $1 \mathrm{~mL}$ PBS solution containing $500 \mu \mathrm{g}$ per $\mathrm{mL}$ of the nanoparticles was gently shaken by hand for 10 minutes and afterwards placed next to a $10 \mathrm{~mm} \mathrm{NdFeB}$ cube magnet (Supermagnete, $1.37 \mathrm{~T}$ ) for 10 seconds. 500 microliters of the supernatant solution was removed, and the iron content was quantified using Inductively Coupled Plasma Mass Spectroscopy (ELEMENT $2^{\mathrm{TM}}$ ICP-MS, Thermo Scientific) in order to determine the separation efficiency. This procedure was repeated for three other separation times, 1, 5 and 20 minutes, respectively.

\section{Bacteria removal}

Methicillin resistant $S$. aureus, among the most prevalent pathogens in human blood, was used as the model bacterium in this study. Bacteria from glycerol stocks were grown on tryptic soy agar (TSA) plates. A single colony was transferred to $10 \mathrm{~mL}$ of $30 \%$ tryptic soy broth (TSB) with $0.25 \%$ glucose and incubated overnight at $160 \mathrm{rpm}$ and $37{ }^{\circ} \mathrm{C}$. The overnight culture was diluted to a final OD600 of 0.01 with fresh $30 \%$ TSB + glucose 
and incubated until it reached an OD600 of 1.0, corresponding to approximately $10^{8}$ colony forming units (cfu) per mL. The cells were harvested via centrifugation at $13000 \mathrm{~g}$ for $5 \mathrm{~min}$. The pellet was washed three times with sterile PBS, and then resuspended in PBS to obtain $10^{3}$ and $10^{4}$ cfu per mL. $500 \mu \mathrm{l}$ of the bacterial suspension was mixed with $50 \mu$ l magnetic beads. Beads without antibody modification and PBS without beads were used as controls. The mixtures were incubated at room temperature on an orbital shaker for $10 \mathrm{~min}$. Beads were collected for $5 \mathrm{~min}$ using an iron neodymium permanent magnet. The supernatant and the collected beads were resuspended in $10 \mathrm{~mL}$ PBS. $200 \mu \mathrm{l}$ of the suspensions were plated on agar plates and incubated overnight at $37^{\circ} \mathrm{C}$.

\section{Bacteria detection and identification}

The above-mentioned collected beads were also utilized for S. aureus DNA purification with GenEluteTM Bacterial Genomic DNA Kit (Sigma-Aldrich). The isolated DNA was further used as the template for in vitro identification and quantification of S. aureus genomes using qPCR. The Primerdesign ${ }^{\mathrm{TM}}$ genesig $^{\circledR}$ Kit (Primerdesign Ltd) containing $S$. aureus specific primers and PrecisionTM Master Mix was used. The primers used in this study allow amplification of femB and have the following sequence: 5'-TTA CAG AGT TAA CTG TTA CC- $3^{\prime}$ and 5'-ATA CAA ATC CAG CAC GCT CT-3'. qPCR was performed according to the manufacturer's instruction.

\section{Particle uptake into monocytic cells}

For particle uptake measurements, particles $\left(10^{10}\right.$ particles per $\left.\mathrm{mL}\right)$ were incubated with human monocyte cells (THP-1 cell line, ECACC 88081201 ) at a concentration of $5 \times 10^{5}$ cells per $\mathrm{mL}$ (equivalent to the monocyte concentration in blood) for 10 minutes, 6 hours and 24 hours, respectively. To detect apoptosis and necrosis induced by the nanoparticles used, the cells were stained using the FITC annexin V Apoptosis Detection Kit II (BD Biosciences). For staining, $5 \mu \mathrm{l}$ of FITC annexin V (component no. 51-65874X) and $5 \mu \mathrm{l}$ PI (component no. 51-66211E) were added to $1 \times 10^{5}$ cells and incubated for 15 minutes. After addition of binding buffer (component no. 51-66121E), the samples were analyzed using flow cytometry with a Gallios Flow Cytometer (Beckman Coulter). The shift of the cell population after nanoparticle incubation was recorded with forward and side scatter. The fluorescence of propidium iodide (PI) and fluorescein isothiocyanate (FITC)-coupled annexin $\mathrm{V}$ was detected in fluorescence channels 1 and 4, respectively. Cadmium sulfate $(15 \mathrm{mM})$ and staurosporine $(0.5 \mu \mathrm{M})$ served as positive controls and were added to the cells $3 \mathrm{~h}$ before cell harvest. Untreated and water treated cells served as the negative controls. To detect possible interferences of the nanoparticles with the assay, the control cells were spiked with the same amount of nanoparticles used in all other samples directly during the staining procedure to ensure no uptake and therefore no biological effect on the cells. Furthermore, nanoparticles incubated for $24 \mathrm{~h}$ in cell culture medium and in water were measured with the same settings in the flow cytometer to ensure no appearance of nanoparticles alone in the forward scatter as well as in the fluorescence channels.

\section{Particle stability and degradation}

The degradation of the nanoparticles was measured at both $\mathrm{pH}$ 4.5 (mimicking lysosomal conditions) and 3.0 (in order to observe a faster degradation) in $10 \mathrm{mM}$ citrate buffer solution. Incubation mixtures were prepared containing $50 \mu \mathrm{g} \mathrm{mL}^{-1}$ of the nanoparticles in a total volume of $2 \mathrm{~mL}$. The rate of degradation of the nanoparticles was measured at different time points (day $1,2,3,6,9,15,21$ ) by measuring the concentration of degraded iron using ICP-MS (ELEMENT $2^{\text {TM }}$ ICP-MS, Thermo Scientific).

\section{Acknowledgements}

The authors acknowledge support from the Novartis Foundation for Medical-biological Research. M. L. acknowledges financial support from the Swiss National Science Foundation, with grant numbers PP00P2133597/1 and PP00P2_159258. N. B. acknowledges funding from the NanoScreen Materials Challenge co-funded by the Competence Centre for Materials Science and Technology (CCMX).

\section{Notes and references}

1 D. C. Angus and T. van der Poll, N. Engl. J. Med., 2013, 369, 840-851.

2 D. C. Angus, W. T. Linde-Zwirble, J. Lidicker, G. Clermont, J. Carcillo and M. R. Pinsky, Crit. Care Med., 2001, 29, 1303-1310.

3 M. J. Llewelyn, M. Berger, M. Gregory, R. Ramaiah, A. L. Taylor, I. Curdt, F. Lajaunias, R. Graf, S. J. Blincko, S. Drage and J. Cohen, Crit. Care, 2013, 17, R60.

4 K.-M. Kaukonen, M. Bailey, D. Pilcher, D. J. Cooper and R. Bellomo, N. Engl. J. Med., 2015, 372, 1629-1638.

5 O. Liesenfeld, L. Lehman, K. P. Hunfeld and G. Kost, Eur. J. Microbiol. Immunol., 2014, 4, 1-25.

6 I. K. Herrmann, M. Urner, F. M. Koehler, M. Hasler, B. RothZ'Graggen, R. N. Grass, U. Ziegler, B. Beck-Schimmer and W. J. Stark, Small, 2010, 6, 1388-1392.

7 I. K. Herrmann, R. E. Bernabei, M. Urner, R. N. Grass, B. Beck-Schimmer and W. J. Stark, Nephrol., Dial., Transplant., 2011, 26, 2948-2954.

8 I. K. Herrmann, M. Urner, S. Graf, C. M. Schumacher, B. RothZ'graggen, M. Hasler, W. J. Stark and B. Beck-Schimmer, Adv. Healthcare Mater., 2013, 2, 829-835.

9 I. K. Herrmann, A. Schlegel, R. Graf, C. M. Schumacher, N. Senn, M. Hasler, S. Gschwind, A.-M. Hirt, D. Guenther, P.-A. Clavien, W. J. Stark and B. Beck-Schimmer, Nanoscale, 2013, 5, 8718-8723.

10 J. H. Kang, M. Super, C. W. Yung, R. M. Cooper, K. Domansky, A. R. Graveline, T. Mammoto, J. B. Berthet, H. Tobin, M. J. Cartwright, A. L. Watters, M. Rottman, A. Waterhouse, A. Mammoto, N. Gamini, M. J. Rodas, A. Kole, A. Jiang, T. M. Valentin, A. Diaz, K. Takahashi and D. E. Ingber, Nat. Med., 2014, 20, 1211-1216. 
11 H. Y. Lee, D. R. Bae, J. C. Park, H. Song, W. S. Han and J. H. Jung, Angew. Chem., Int. Ed., 2009, 48, 1239-1243.

12 L. Wang, Z. Yang, J. Gao, K. Xu, H. Gu, B. Zhang, X. Zhang and B. Xu, J. Am. Chem. Soc., 2006, 128, 13358-13359.

13 J. H. Kang, E. Um, A. Diaz, H. Driscoll, M. J. Rodas, K. Domansky, A. L. Watters, M. Super, H. A. Stone and D. E. Ingber, Small, 2015, 11, 5657-5666.

14 I. K. Herrmann, B. Beck-Schimmer, C. M. Schumacher, S. Gschwind, A. Kaech, U. Ziegler, P.-A. Clavien, D. Günther, W. J. Stark, R. Graf and A. A. Schlegel, Nanomedicine, 2016, 11, 783-796.
15 W. J. Stark, Angew. Chem., Int. Ed., 2011, 50, 1242-1258.

16 C. Cywes-Bentley, D. Skurnik, T. Zaidi, D. Roux, R. B. DeOliveira, W. S. Garrett, X. Lu, J. O’Malley, K. Kinzel, T. Zaidi, A. Rey, C. Perrin, R. N. Fichorova, A. K. K. Kayatani, T. Maira-Litràn, M. L. Gening, Y. E. Tsvetkov, N. E. Nifantiev, L. O. Bakaletz, S. I. Pelton, D. T. Golenbock and G. B. Pier, Proc. Natl. Acad. Sci. U. S. A., 2013, 110, E2209-E2218.

17 T. Rimmelé and J. A. Kellum, Crit. Care, 2011, 15, 205.

18 H. Suzuki and H. Hirasawa, Acute Blood Purification, Karger Medical and Scientific Publishers, 2010. 